\title{
A simple method for potential flow simulation of cascades
}

\author{
ARAVIND BHIMARASETTY and RAGHURAMAN N \\ GOVARDHAN*
}

Department of Mechanical Engineering, Indian Institute of Science, Bangalore 560012

e-mail: raghu@mecheng.iisc.ernet.in

MS received 21 April 2009; revised 1 February 2010; accepted 23 August 2010

\begin{abstract}
A simple method using a combination of conformal mapping and vortex panel method to simulate potential flow in cascades is presented. The cascade is first transformed to a single body using a conformal mapping, and the potential flow over this body is solved using a simple higher order vortex panel method. The advantage of this method over existing methodologies is that it enables the use of higher order panel methods, as are used to solve flow past an isolated airfoil, to solve the cascade problem without the need for any numerical integrations or iterations. The fluid loading on the blades, such as the normal force and pitching moment, may be easily calculated from the resultant velocity field. The coefficient of pressure on cascade blades calculated with this methodology shows good agreement with previous numerical and experimental results.
\end{abstract}

Keywords. Cascade; turbo-machinery; aerodynamics; conformal mapping.

\section{Introduction}

Potential flow in cascades is a well-studied problem. Gostelow (1984) gives reference to several sources in the literature where such studies have been undertaken. Broadly, the problem has been solved by using either a sequence of conformal transformations or by using surface singularity methods; the latter also referred to as panel methods in the fluid dynamics community (Hess 1990).

In the sequence of conformal transformations method, the infinite row of blades in the physical plane is first transformed to a single closed contour. Thereafter a series of transformations are used to reduce this shape to a circle. For example, Howell (1948) used the $\varsigma=\tanh (z)$ transformation to map a linear cascade to an S-shaped closed contour followed by two or more Joukowski transformations to further transform the S-shaped closed contour to an irregular circle. Thereafter, Theodorsen's iterative procedure (Theodorsen 1931) was used to arrive at a regular circle. Garrick (1944), Traupel (1945) have used different mappings to reduce the linear cascade to a single closed shape after which it was transformed to a regular circle using Theodorsen's method (Theodorsen 1931). However, this process is

*For correspondence 
iterative with the number of iterations dependent on the shape of the blades and is in general not very amenable for programming on a computer.

Surface singularity methods, which can be easily programmed, employ an alternate route to solve the potential flow problem in cascades by distributing singularities such as vortices and/or sources on the blade contour. Due to the geometry of the infinite cascade, every singularity on a portion of one cascade blade has to be repeated an infinite number of times, corresponding to each one of the cascade blades. The induced velocity due to this infinite array of singularities with a regular spacing can be summed up and represented by one term, as given for example in Gostelow (1984) and Lewis (1991). The potential flow through the cascade can then be represented by a number of such infinite array singularities placed along the blade surface. Such surface singularity methods using discrete point vortices/sources were developed by Birnbaum (1923), Glauert (1924) and Allan (1962). It may be noted here that the solution methodology for cascades given in Lewis (1991) also in effect involves the placing of such discrete infinite array singularities along the blade surface. A higher order version of this method, using continuously distributed sources/vortices was formulated by McFarland (1982). However, in this method, the integration for the effect of the continuously distributed infinite array of vortices on a panel involves complicated expressions and has to be done numerically. This is in contrast to simple higher order panel methods, as is used to solve the isolated airfoil problem, where the integrals across a panel for the simpler single vortex can be done analytically, as shown for example by Kuethe \& Chow (1986).

The methodology proposed in this paper offers a simple way to solve the potential flow through a cascade problem by using a combination of a single conformal transformation and a standard higher order vortex panel method such as the one used to solve the potential flow past an isolated airfoil. The main advantage with the current method is that a higher order panel code from an aerodynamics text-book like Kuethe \& Chow (1986) may be directly used to solve the cascade problem without the need for any numerical integration across the panel, as required in the methodology of McFarland (1982), or the requirement of iterations as in a number of other methods. These advantages should make the present methodology faster and easier to implement. Also, as discussed in (Hess 1990), the use of a higher order panel method should result in better accuracies when individual elements (blades) are placed close together.

It may be noted that a more involved combination method utilizing conformal transformation and surface singularities was more recently proposed by Tanaka et al (1991), for the purpose of improving the accuracy of the predicted pressure distribution $\left(C_{p}\right)$ over thin blades or blades with cusped trailing edge in cascades. In their method, the row of airfoils was transformed into a row of irregular circles using an iterative procedure. Singularity method was then used to solve the potential flow past this cascade of irregular circles.

\section{Methodology}

The simple method proposed here works for any infinite linear cascade composed of arbitrary shaped blades separated by a uniform spacing $h$. Irrespective of the blade shape, spacing or stagger, the method will solve the problem without the need for any iterations or numerical integrations. Figure 1 shows an example infinite linear cascade. The physical plane that contains this geometry will henceforth be called as the $z_{1}$ plane.

It is well known that the potential flow past an isolated airfoil can be easily solved using high order vortex panel methods, where the airfoil is discretized into panels with each panel consisting of a continuous vortex sheet whose strength has to be determined. The 


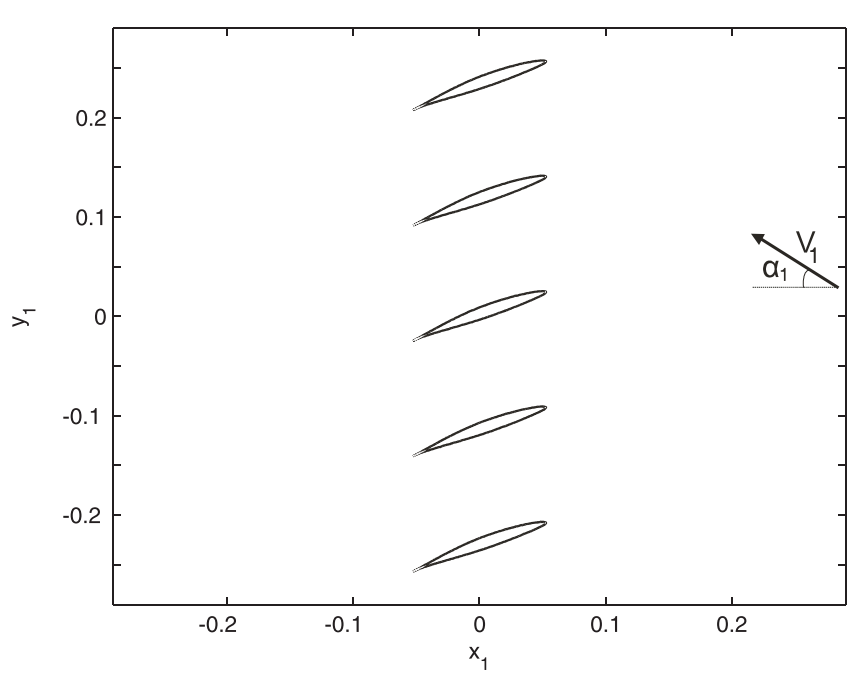

Figure 1. Example linear cascade of NACA 65410 blades in the physical $z_{1}$-plane. In the figure, $V_{1}$ and $\alpha_{1}$ are the inlet flow velocity and inclination far upstream of the cascade.

no-penetration conditions on each panel, and the Kutta condition at the trailing edge, provide a complete set of linear equations with which the strength of the vortex sheet on each panel can be determined, as discussed by Hess (1990). It should be noted that the induced velocity at an arbitrary point due to the continuous vortex sheet on a panel, can be obtained in closed form analytical expressions, and hence no numerical integrations across the panel are required.

The idea of the current method is to directly utilize the standard higher order vortex panel method to solve the cascade problem by first utilizing a conformal mapping to transform the infinite cascade to a single closed contour. By doing so, we avoid the series of transformations that are usually employed in pure conformal transformation methods in order to reduce this single closed contour to a regular circle. We are also able to use the panel code of the isolated airfoil problem to solve the cascade problem without the need for any numerical integrations, as required in higher order surface singularity methods like that of McFarland (1982).

\subsection{Conformal transformation}

For the single conformal transformation, the mapping given by Howell (1948) and Thwaites (1987) is used: $z_{2}=\tanh \left(\frac{\pi z_{1}}{h}\right)$, where $h$ is the spacing between blades in the linear-cascade in the physical $z_{1}$-plane. Once this transformation is applied, all the blades in the linear cascade system shown in the $z_{1}$-plane are transformed into a single contour in the $z_{2}$-plane, as shown in figure 2 for the example linear cascade.

Regarding the flow, as explained in Thwaites (1987), the region far upstream of the cascade in the $z_{1}$-plane is mapped on to the point $(+1,0)$ in the $z_{2}$-plane, and hence the incoming flow in the $z_{1}$-plane may be represented in the $z_{2}$-plane by a combination of a point source and a point vortex at $(+1,0)$. The point source of strength $Q=V_{1} h \cos \left(\alpha_{1}\right)$ represents the flow rate between two blades that is dependent on the horizontal velocity component $\left(V_{1} \cos \alpha_{1}\right)$, while the vertical velocity component $\left(V_{1} \sin \alpha_{1}\right)$ is represented by a point vortex, $\Gamma_{\text {upstream }}=V_{1} h \sin \left(\alpha_{1}\right)$, where $V_{1}$ and $\alpha_{1}$ are the inlet flow velocity and angle of inclination far upstream of the cascade. Similarly, the exit conditions $($ at $-\infty)$ in the $z_{1}$-plane can be represented by a combination of a sink $(-Q)$ and a vortex $\left(\Gamma_{\text {downstream }}\right)$ located at $(-1,0)$ in the $z_{2}$-plane. It should be noted here that mass conservation in the $z_{1}$-plane requires the sink magnitude to be the same as that of the source, while the downstream vertical velocity 


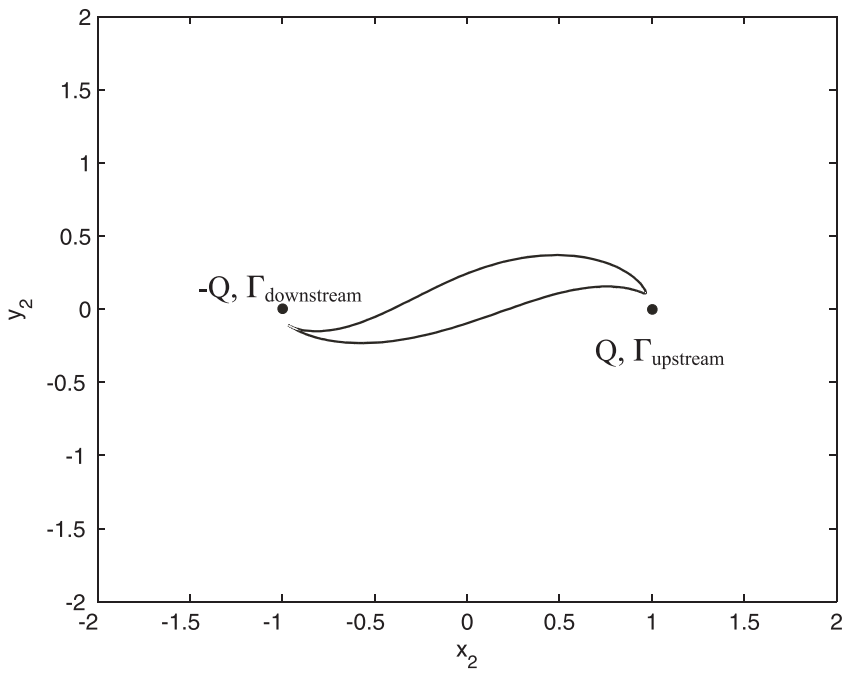

Figure 2. Transformed single contour in the $z_{2}$-plane using the conformal mapping: $z_{2}=$ $\tanh \left(\frac{\pi z_{1}}{h}\right)$, for the example case shown in figure 1. The far upstream and downstream velocities are represented in this plane by the point source/sink of strength $\mathrm{Q}$ and the point vortices $\left(\Gamma_{\text {upstream }}, \Gamma_{\text {downstream }}\right)$ at the locations indicated by the filled in circles.

component, represented by the point vortex $\left(\Gamma_{\text {downstream }}\right)$ in the $z_{2}$-plane, will change from the upstream value and needs to be determined, as explained in the next sub-section.

The potential flow around the single closed contour in the $z_{2}$-plane in the presence of the source $(Q)$ and vortex $\left(\Gamma_{\text {upstream }}\right)$ at $(+1,0)$ and the sink $(-Q)$ and the unknown vortex $\left(\Gamma_{\text {downstream }}\right)$ at $(-1,0)$ may be solved using the vortex panel method, which is briefly described below.

\subsection{Vortex panel method}

The single closed contour lying in the $z_{2}$-plane is split up in to a large number of discrete panels. This is illustrated in figure 3 that shows the $(m+1)$ nodal points representing the $m$ panels, with the nodal vorticity strengths for an arbitrary $k^{\text {th }}$ panel of length $l_{k}$ being given by $\gamma_{k}$ and $\gamma_{k+1}$. For the panel method, we use a higher order vortex panel method having a linear variation of vorticity within the panel, as given in (Kuethe \& Chow 1986), and shown in the

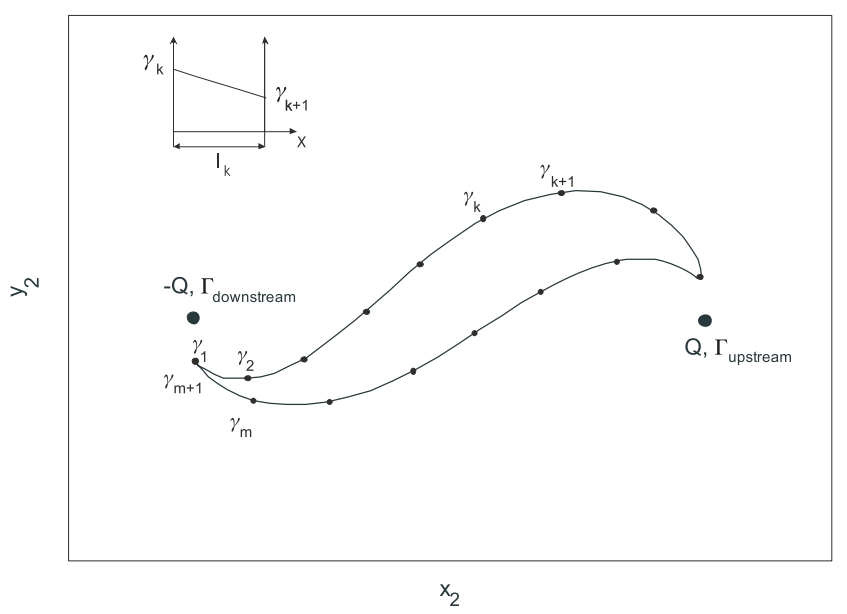

Figure 3. This figure illustrates the discretization of the transformed single contour in to $m$ vortex panels with $m+1$ nodes. The vorticity distribution along each panel is linear as shown in the inset. The unknowns for each calculation are the $(m+1)$ nodal vorticity strengths $\left(\gamma_{1}, \gamma_{2}, \ldots \gamma_{m+1}\right)$ and $\Gamma_{\text {downstream }}$ that represents the unknown vertical velocity component far downstream of the cascade. 
inset of figure 3. In this panel method, the number of unknowns in the form of nodal vorticity strengths is equal to one more than the number of panels $(m)$. In our problem, the vorticity at the sink location $\left(\Gamma_{\text {downstream }}\right)$ is an additional unknown, which effectively represents the unknown vertical velocity component far downstream of the cascade. Therefore, there are $(m+2)$ unknowns and the same number of equations are required to solve for the unknowns. As usual, the no-penetration condition at the control points on the panels and the Kutta condition at the trailing edge yield $(m+1)$ equations. It may be noted that the form of Kutta condition used for the present calculations is the zero loading condition, i.e. $\gamma_{1}=\gamma_{m+1}$.

The extra $(m+2)$ equation is given by the fact that in the $z_{1}$-plane, the change in vertical velocity from far upstream to far downstream of the cascade should be equal to the circulation around one blade. This is explained in Thwaites (1987) and also given below for the sake of clarity.

Consider the net circulation around the path $a-b-c-d$, in figure 4, where the parallel segments $b-c$ and $a-d$ represent streamlines and the segments $a-b$ and $c-d$ are straight line segments far upstream and far downstream respectively of the cascade. The net circulation around the path $\mathrm{a}-\mathrm{b}-\mathrm{c}-\mathrm{d}$ is the circulation around one blade, $\Gamma_{\text {blade }}$, and is given by:

$$
\Gamma_{\text {blade }}=h\left(V_{1} \sin \alpha_{1}+V_{2} \sin \alpha_{2}\right),
$$

where $\alpha_{1}$ and $\alpha_{2}$ are angles that $V_{1}$ and $V_{2}$ make with the horizontal and $\Gamma_{\text {blade }}$ is positive anticlockwise. The above relation results due to the fact that the velocity field is periodic in the $z_{1}$ plane, and therefore $\int_{b}^{c} \mathbf{v} \cdot \mathbf{d s}=-\int_{d}^{a} \mathbf{v} \cdot \mathbf{d s}$. Hence, in the circulation expression, only the integral for paths a-b and c-d remain. Noting that in the $z_{2}$-plane, $\Gamma_{\text {downstream }}=V_{2} h \sin \left(\alpha_{2}\right)$, we may re-write eq. (1) as:

$$
\Gamma_{\text {blade }}-\Gamma_{\text {downstream }}=V_{1} h \sin \alpha_{1} .
$$

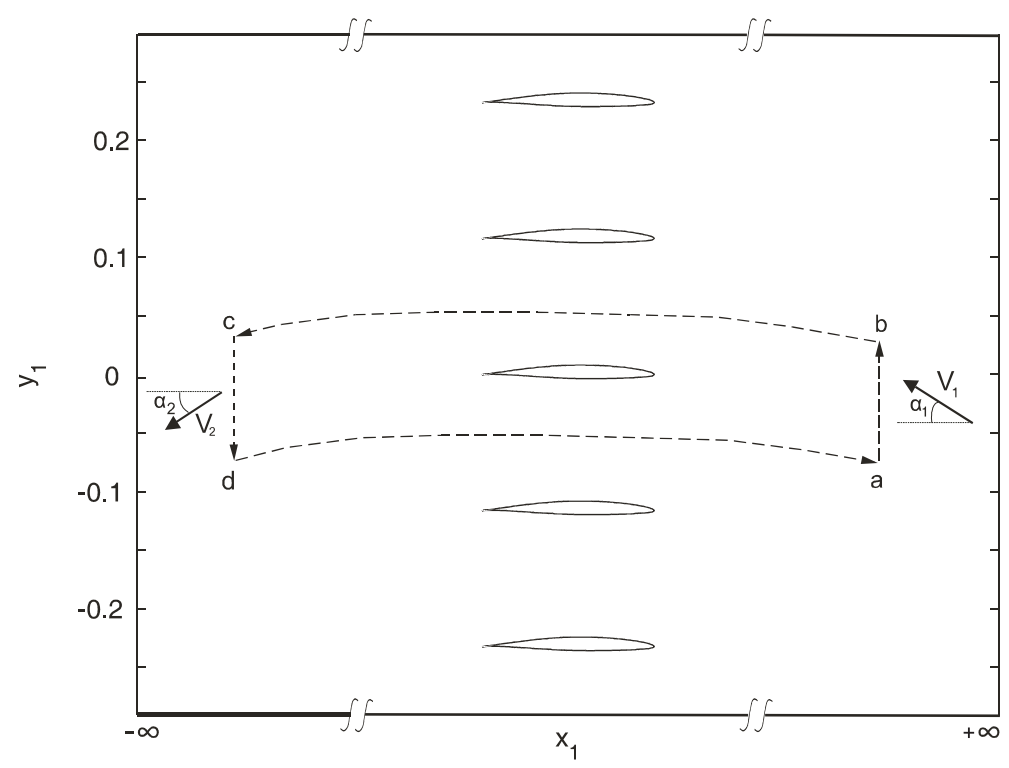

Figure 4. Path $a-b-c-d$ in the physical $z_{1}$-plane along which the circulation is calculated. The flow here is from right to left, with the path $\mathrm{a}-\mathrm{b}$ corresponding to the far upstream conditions and the path $\mathrm{c}-\mathrm{d}$ corresponding to the far downstream condition. 

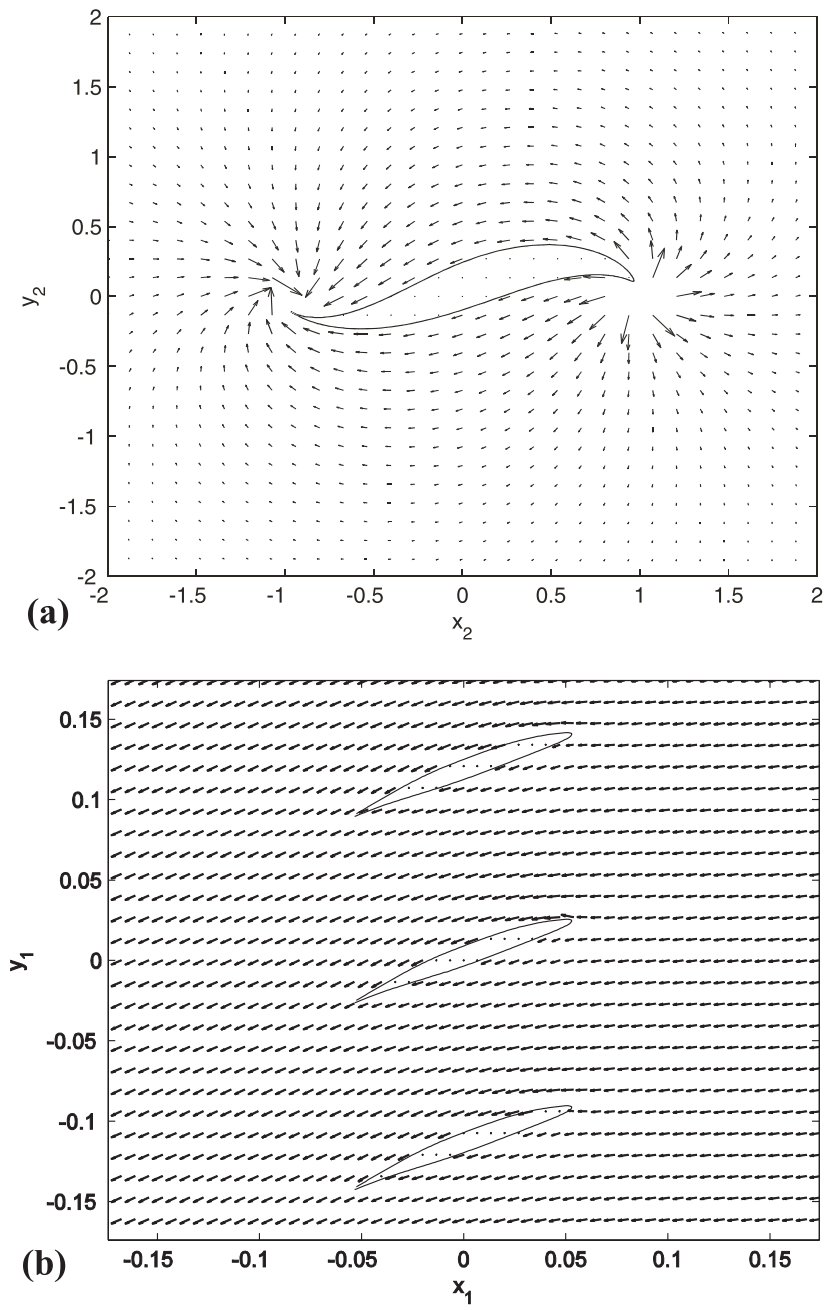

Figure 5. In (a), the calculated potential velocity field in the $z_{2}-$ plane is shown for the example case in figure 1. In (b), the transformed velocities in the physical $z_{1}$-plane are shown.

This equation provides the expression for $\Gamma_{\text {downstream }}$, since the inlet conditions $V_{1}$ and $\alpha_{1}$ are known and $\Gamma_{\text {blade }}$ can be easily expressed in terms of the summation of vorticity of the panels (noting that the vorticity is linearly varying along each panel, $\Gamma_{\text {blade }}=\sum_{k=1}^{m} \frac{\left(\gamma_{k}+\gamma_{k+1}\right) l_{k}}{2}$ ). This equation along with the no-penetration and Kutta condition is sufficient to solve the potential flow problem in the $z_{2}$-plane using a simple higher order vortex panel method code such as the one in (Kuethe \& Chow 1986) without the need for any numerical integrations. Only minor modifications need to be made to the standard panel method code to include the effects of the extra known point source, sink and $\Gamma_{\text {upstream }}$, and the unknown point vortex, $\Gamma_{\text {downstream }}$.

Once the potential flow is solved in the $z_{2}$-plane using the panel method, the magnitude of the velocities on the contour may be easily transformed to obtain the magnitudes of the velocities on the cascade blades in the physical $z_{1}$-plane. Since the planes $z_{1}$ and $z_{2}$ are related by a conformal transformation, the relation between their velocities is given by:

$$
u_{1}-i v_{1}=\left(u_{2}-i v_{2}\right) \frac{d z_{2}}{d z_{1}}
$$




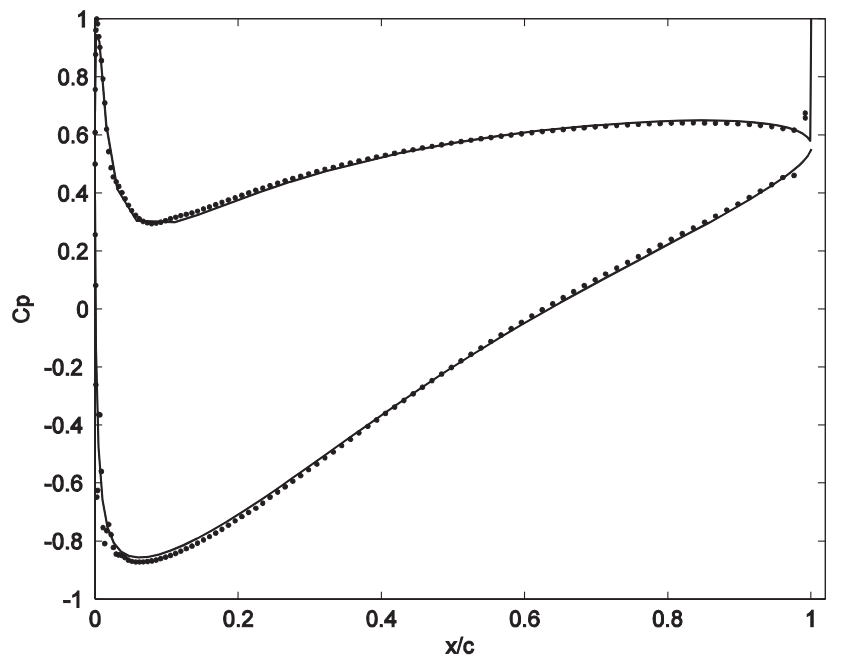

Figure 6. Comparison of coefficient of pressure $\left(C_{p}\right)$ from the present methodology with the exact results given in Gostelow (1984) for a cusped blade. - present method, - Gostelow (1984) [for stagger $(\beta)=37 \cdot 5^{\circ}$, solidity $(\sigma)=$ 1.01 and $\alpha_{1}=53 \cdot 5^{\circ}$.

where $i$ is $\sqrt{-1}, u_{1}-i v_{1}$ and $u_{2}-i v_{2}$ are the complex velocities in the $z_{1}$ and $z_{2}$-planes respectively.

\section{Results}

This method has been programmed so that for a user-defined set of input values, the program plots the velocity fields and pressure distribution on the blades and computes the fluid loading on the blade, such as the lift force and pitching moment, from the pressure distribution. Figure 5a shows the computed velocity field in the $z_{2}$-plane for the cascade of figure 1 , and figure $5 \mathrm{~b}$ the corresponding transformed velocities in the physical $z_{1}$-plane, for a set of input parameters.

In order to numerically validate the present code, the coefficient of pressure $\left(C_{p}\right)$ was calculated for a cascade whose exact pressure distribution is given in Gostelow (1984). The agreement between the two sets of results is good, as can be seen from figure 6 .

Comparison of the coefficient of pressure $\left(C_{p}\right)$ was also made with experimental results from Emery et al (1958), who have given $C_{p}$ variations for NACA 65410 profiles for various stagger angles $\left(\beta_{1}\right)$, solidity $(s)$, and angles of attack $\left(\alpha_{1}\right)$. Two of these cases are shown in figure 7 along with the results from the present calculations. As seen from these figures, the present methodology is able to predict well the experimental data.

Although only linear cascades have been discussed here, it should be noted that the current method can also be easily extended to calculate the potential flow in circular cascades. This can be done by first using an additional conformal transformation, $\varsigma=r_{c} \log z_{1}$, to transform the circular cascade into a linear cascade, and then proceeding in a similar manner.

\section{Conclusion}

A simple method that uses a combination of conformal mapping and a simple higher order panel method to solve the potential flow in cascades is presented. With this method, the potential flow in linear and circular cascades may be easily calculated with the use of simple higher 

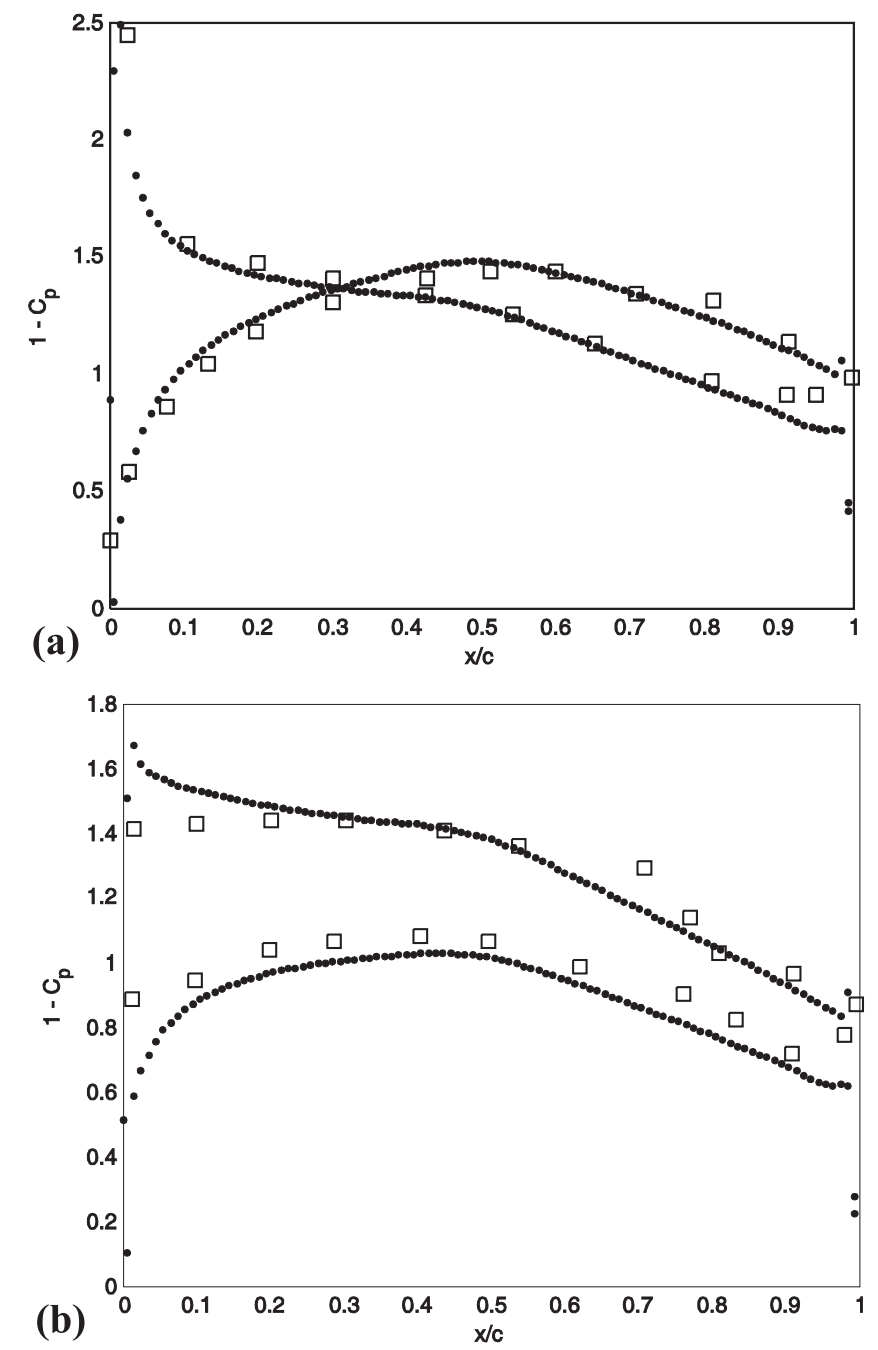

Figure 7. Comparison of pressure distribution between present method and experiments. - Present method and $\square$ Experimental measurements from (Emery et al 1958). In (a), the stagger $\beta_{1}=30^{\circ}$, solidity, $\sigma=1$ and $\alpha_{1}=-3^{\circ}$, while in (b) the stagger $\beta_{1}=30^{\circ}$, solidity, $\sigma=1$ and $\alpha_{1}=9^{\circ}$.

order panel method, as is used for isolated airfoils, combined with a single conformal transformation. The advantage of the proposed simple method is that it enables the use of higher order vortex panel methods that are considered more accurate, for the cascade problem without the requirement of numerical integrations. Comparisons of experimental and analytical results are presented, which demonstrate that the present method predicts the pressure distributions very well.

The methodology may prove useful for rapid calculation of forces and moments on linear and circular cascade blades, and in the design of cascade blade geometries. The potential flow solutions for circular cascades may also offer some insight into the flow past guide vanes in centrifugal compressors and turbines. Since the current potential flow code is very fast when compared with a full Navier-Stokes solution, it may be used in inverse design problems of cascades using a heuristic method such as the genetic algorithm. It may also find application in simulation of unsteady cascade problems. 


\section{References}

Allan W K A theory of cascade interference. ARC, 23, 214

Birnbaum W 1923 Die tragende wirbelfläche hilfsmittel zur behandlung desebenen problems der traflugelthoerie. ZAMM 3(4): 290

Emery J C, Herrig L J, Erwin J R, Felix A R 1958 Systematic two-dimensional cascade tests of NACA 65-series compressor blades at low speeds. NACA-TR-1368

Garrick I 1944 On the plane potential flow past a lattice of arbitrary aerofoils. NACA Report 788

Glauert H 1924 A theory of thin aerofoils. ARC R\& M, No. 910

Gostelow J P 1984 Cascade aerodynamics. Pergamon Press

Hess J L 1990 Panel methods in Computational fluid dynamics. Annual Rev. of Fluid Mech. 22: 255-274

Howell A 1948 The theory of arbitrary aerofoils in cascades. Phil. Mag. 39: 299, 913-927

Kuethe A M, Chow C-Y 1986 Foundations of aerodynamics. Wiley

Lewis R I 1991 Vortex element methods for fluid dynamic analysis of engineering systems. Cambridge University Press

McFarland E R 1982 Solution of plane cascade flow using improved surface singularity methods. J. Eng. for Power 104(3):

Tanaka S, Murata S, Kurata K 1991 Computation of the potential flow through cascades using the conformal mapping and the singularity method. JSME Int. Journal Series II 34(4):

Theodorsen T 1931 Theory of wing sections of arbitrary shape. NACA Report 411

Thwaites B 1987 Incompressible aerodynamics. Dover

Traupel W 1945 Die berechnung der strömung durch schaufelgitter. Sulzer Tech. Rundschau 1: 25-42 